INPLASY PROTOCOL

To cite: Zhang et al. Updated evidence on the antioxidant effects of coenzyme Q10 supplementation: a various population data meta-analysis. Inplasy protocol 2021120123. doi:

10.37766/inplasy2021.12.0123

Received: 27 December 2021

Published: 27 December 2021

Corresponding author:

Yili Zhang

zhangyili823@126.com

Author Affiliation:

Nanjing University of Chinese Medicine.

Support: None.

Review Stage at time of this submission: Preliminary searches.

Conflicts of interest: None declared.

\section{Updated evidence on the antioxidant effects of coenzyme Q10 supplementation: a various population data meta-analysis}

Zhang, YL1; Huang, XY2; Liu, N3; Liu, MM4; Sun, CR5; Qi, BY6; Sun $\mathrm{K}^{7}$; Wei, $\mathrm{X}^{8}$; Ma, Y9; Zhu, LG ${ }^{10}$.

Review question / Objective: This study aim to systematically summarize randomized clinical trials which have evaluated the impacts of Coenzyme Q10 on oxidative stress biomarkers. Condition being studied: Oxidative stress plays an important role in the occurrence of chronic diseases. Oxidative stress biomarkers are involved in the natural development of multiple diseases, such as cancer, Alzheimer's disease, and myocardial infarction. These biomarkers represent the imbalance amongst reactive oxygen species (ROS) and biological components to detoxify metabolic wastes as well as to address potential cell injury.

Information sources: Comprehensive computerized systematic searches wll be performed throughout PubMed/ Medline, Web of science, Science direct, EMBASE, Cochrane Central Register of Controlled Trials, Chinese National Knowledge Infrastructure(CNKI), and Chinese Biomedical Literature Database (CBM) from inception until Sept. 2021. In addition, we will review the reference lists of eligible reports, previous systematic reviews, guidelines, and will contact industry representatives to identify additional studies.

INPLASY registration number: This protocol was registered with the International Platform of Registered Systematic Review and Meta-Analysis Protocols (INPLASY) on 27 December 2021 and was last updated on 27 December 2021 (registration number INPLASY2021120123).

\section{INTRODUCTION}

Review question / Objective: This study aim to systematically summarize randomized clinical trials which have evaluated the impacts of Coenzyme Q10 on oxidative stress biomarkers.
Condition being studied: Oxidative stress plays an important role in the occurrence of chronic diseases. Oxidative stress biomarkers are involved in the natural development of multiple diseases, such as cancer, Alzheimer's disease, and 
myocardial infarction. These biomarkers represent the imbalance amongst reactive oxygen species (ROS) and biological components to detoxify metabolic wastes as well as to address potential cell injury.

\section{METHODS}

Participant or population: Adult people ( $\geq$ 18 years old).

Intervention: Coenzyme Q10.

Comparator: Placebo.

Study designs to be included: Randomized controlled trials.

Eligibility criteria: Studies will be included if they: 1) are randomized controlled trials with parallel design; 2) are performed on adult people ( $\geq 18$ years old), and 3 ) assess the effects of oral coenzyme Q10 supplementation on aforementioned OS biomarkers compared with the placebo. No restrictions will be applied based on the baseline health status, sex, race of the participants, and date.

Information sources: Comprehensive computerized systematic searches wll be performed throughout PubMed/Medline, Web of science, Science direct, EMBASE, Cochrane Central Register of Controlled Trials, Chinese National Knowledge Infrastructure(CNKI), and Chinese Biomedical Literature Database (CBM) from inception until Sept. 2021. In addition, we will review the reference lists of eligible reports, previous systematic reviews, guidelines, and will contact industry representatives to identify additional studies.

Main outcome(s): Oxidative stress biomarkers.

Quality assessment / Risk of bias analysis: The methodological quality of trials will be assessed independently using criteria from the Cochrane Handbook for Systematic Review of Interventions, Version 5.3.0.
Strategy of data synthesis: Revman $\mathbf{5 . 3}$ software provided by the Cochrane Collaboration will be used for data analyses. The model will be used to pool the data depends on the existence and extent of heterogeneity. If the 12 statistics are less than $50 \%$, the heterogeneity could be accepted, the fixed-effect model will be chosen. While random-effects model will be chosen if the 12 statistics exceeded $50 \%$. The random-effect model will also be used when subgroup analysis is adopted and heterogeneity among studies is obvious. For dichotomous the pooled relative risk (RR) with $95 \%$ confidence interval (Cl) will be used as the effect measure. For the continuous outcomes, weighted mean difference (WMD) or standard mean difference (SMD) will be used as the effect measure. If the number of included studies is less than two or heterogeneity is apparent, meta-analysis is neither appropriate nor possible. Under those circumstances, the result of our systematic review will be narratively reported. If included studies have multiple arms, we will identify the relevant intervention and control groups and combine the relevant intervention groups into a single group, and the relevant control groups into a single group, before synthesizing the data.

Subgroup analysis: A subgroup metaanalysis comparing studies with different dosages, duration, and types of diseases will be conducted.

Sensitivity analysis: Sensitivity analysis will be conducted to detect the impact of each study on the pooled effect size using the leave-one-out method.

Country(ies) involved: China.

Keywords: evidence-based medicine; Coenzyme Q10; oxidative stress; biomarker

Contributions of each author:

Author 1 - Yili Zhang.

Author 2 - Xinyi Huang.

Author 3 - Ning Liu.

Author 4 - Mengmin Liu.

Author 5 - Chuanrui Sun. 
Author 6 - Baoyu Qi.

Author 7 - Kai Sun.

Author 8 - Xu Wei.

Author 9 - Yong Ma.

Author 10 - Liguo Zhu. 